\title{
How Breastfeeding Behavior is Affected by the Breastfeeding Perspectives of Fathers in Georgia (USA)
}

\author{
Les'Shon Irby, MPH ${ }^{1}$, Emily Graybill, PhD, NCSP ${ }^{1}$, and Cassandra White, $\mathrm{PhD}^{1}$ \\ ${ }^{1}$ Georgia State University. Atlanta, GA
}

Corresponding Author: Les'Shon Irby • 404-423-8640 • LesShon.Irby@outlook.com

\begin{abstract}
Background: Father support has recently been associated with increasing breastfeeding rates, but research is limited on how the American father's perspectives of breastfeeding influence breastfeeding behavior. The aim of the study was to investigate the perspectives of fathers residing in Georgia on breastfeeding and to understand if it contributed to mothers' decisions to breastfeed.
\end{abstract}

Methods: We conducted 10 semi-structured, in-depth interviews with parents of ever-breastfeed infants who were at least six months of age.

Results: Mothers often led the decision-making process to breastfeed based on her prepartum knowledge of breastfeeding. Many fathers had no prepartum opinion on breastfeeding, and their lack of opinion was not a determining factor in a mother's decision to initiate and continue exclusive breastfeeding. Fathers generally developed a favorable opinion of breastfeeding due to the perceived benefits their child(ren) received. Data also suggested that reasons for supplementing or transitioning to formula were not associated with the father's perspective of breastfeeding.

Conclusions: Although the father's perspective on breastfeeding had minimal impact on the mother's decision to breastfeed, having the father's support during breastfeeding aided mothers on their breastfeeding journey and with everyday care taking.

Keywords: Breastfeeding behavior, breastfeeding support, exclusive breastfeeding, father, qualitative methods

\section{INTRODUCTION}

Breastfeeding rates worldwide are slowly on the rise (Cai, Wardlaw, \& Brown, 2012). Up by $16.7 \%$ since 1995, in 2010 $39 \%$ of infants were exclusively breastfed for at least six months (Cai et al., 2012). This increase has been associated with the many benefits breastfeeding offers infants and mothers. Consumption of human milk is related to improved infant mortality rates, nutrition, development, and overall health reducing the risk of otitis media, upper and lower respiratory tract infection, sudden infant death syndrome (SIDS), RSV bronchiolitis, ear infection, and inflammatory bowel disease (AAP, 2015; UNICEF, 2015). Mothers who initiate breastfeeding immediately after delivery experience a reduction in the risk of various post- partum conditions like post-partum hemorrhage, depression, type 2 diabetes, and breast/uterine/ovarian cancer; continuation of breastfeeding delays the return of fertility and quick return of pre-pregnancy weight (AAP, 2015; UNICEF, 2015).

Despite health benefits, the United States (US) still has some of the lowest breastfeeding rates internationally. With a national "ever breastfed rate" of $81.1 \%$, the US rate of breastfeeding decreases as infant age increases. The World Health Organization (WHO), United Nation Children's Fund (UNICEF), the American Academy of Pediatrics (AAP) and the American Academy of Family Physicians (AAFP) recommends exclusive breastfeeding for the first 6 months after birth; continued breastfeeding while gradually introducing nutritionally adequate, age appropriate, solid foods beginning at six months of age (WHO, 2011; UNICEF, 2018; AAP, 2015; AAFP, 2017). The 2016 U.S. Breastfeeding Report Card says by three months of age only $44.4 \%$ of infants are exclusively breastfed, and by six months this rate decreases to $22.3 \%$ (CDC, 2016). Similarly, in the state of Georgia the "ever breastfed rate is $69.2 \%$ with only $38.7 \%$ being exclusively breastfeed by three months of age, and $25.4 \%$ by six months.

So why does breastfeeding cessation often take place during a child's first year of life in the US? Research has shown that lack of knowledge, lactation challenges, nutrition concerns, low family/social support, embarrassment, and barriers associated with employment and childcare are common reasons for breastfeeding cessation (Ahluwalia, Morrow, \& Hsia, 2005; Li, Fein, Chen, \& Grummer-Strawn, 2008; Office of the Surgeon General, 2011). 
There are many factors that influence the decision to breastfeed, including the infant health benefits, naturalness, emotional bonding, and social support (Shahla, Fahy, \& Kable, 2010; Arora, Wehrer, \& Kuhn, 2000). There is an association between longer durations of exclusive breastfeeding and adequate family support, specifically strong approval from the infant's father for the mother to breastfed (Chang \& Chan, 2003; Cernadas, Graciela, Barrera, Martinez, \& Garsd, 1994). Though mothers' attitudes and beliefs can have a major influence, partner support has been determined to play an important role in the decision to initiate and continue breastfeeding including exclusivity (Mueffelmann, Racine, Warren-Findlow, \& Coffman, 2014; Sharma \& Petosa, 1997). Studies have shown that a mother's intention to breastfeed was positively influenced by a partner's preference and that a lack of partner support was negatively associated with a mother's intention to breastfeed (Mannion, Hobbs, McDonald, \& Tough, 2013; Kessler, Gielen, Diener-West, \& Paige, 1995; Freed, Gary, \& Fraley, 1993).

Though studies have suggested that support of fathers may be associated with increased length of breastfeeding, more research is needed on whether fathers' views of breastfeeding affect the initial decision to breastfeed. The aim of this study is to investigate the perspective Georgia fathers have on breastfeeding and understand if their perspective contributes to mothers' decisions to initiate and continue exclusive breastfeeding for at least six months.

\section{METHODS}

\section{Research questions}

The following research questions will be explored in this study.

1. What are the perceptions Georgia fathers have about breastfeeding?

2. What has influenced the father's perception(s) about breastfeeding?

3. Do fathers' perception about breastfeeding relate to the mother's decision to initiate and continue breastfeeding?

\section{Participants}

The research described in this manuscript was approved by our university's Institutional Review Board. Participants were recruited via flyers posted at a large urban university in the southeastern region of the US and a 501(c) 3 non- profit organization that provides lactation and nutrition support. To broaden recruitment, flyers were also distributed via email among personal and professional contacts. Inclusion criteria were to: 1) be a heterosexual couple of a child at least six months of age, 2) have a child that was ever-breastfed, 3) be at least 18 years of age, and 4) have both parents available to be interviewed separately. Eleven couples contacted the research team; eight couples were eligible; and five couples completed the study. Respondents were asked to report their age, race, and highest level of education completed.
Recruitment took place across four months and continued until data saturation was achieved. Couples who were interested in the study could contact the research team via telephone or email to determine eligibility. In-depth interviews (IDIs) were conducted across four in private spaces that were convenient to the participant. Informed consent and a demographic questionnaire was obtained from each participant.

\section{Data collection and analysis}

Separate interview protocols were created for mothers and fathers to guide each IDI. Each IDI was completed in less than 60 minutes. NVivio 9 was used for data organization. Guided by principles of grounded theory, data were open coded for common themes. During axial coding, common themes were collapsed and reorganized according to category of themes. The mothers' and fathers' interviews were coded separately and then common codes across subgroups were identified.

\section{RESULTS}

Table 1 shows participant demographic characteristics. The sample consisted of five couples (10 participants) whose ages ranged from 30 to 44 years of age (Table 1). The majority of the participants were African American (50\%), with others being identified as White $(20 \%)$, Filipino (20\%), and Hispanic (10\%) (Table 1). $70 \%$ of the participants identified having an associate's degree or higher (Table 1). All participants reported being married (Table 1).

Results suggested that mothers served as the lead decision makers in the choice to breastfeed their child(ren), while fathers deferred to what their partner decided and supported that decision. Fathers who did not have a prepartum perspective of breastfeeding developed a favorable opinion over time. When partners spoke about breastfeeding cessation, supplementation, or the transition to infant formula, the decision to transition was influenced by professional barriers to breastfeeding rather than lack of father support. 


\begin{tabular}{|l|c|c|}
\hline \multicolumn{1}{|c|}{ Characteristic } & Mothers N=5 \\
\hline Age Group & 1 & 2 \\
$30-34$ & 2 & 3 \\
$35-39$ & 2 & 3 \\
$40-44$ & 2 & 1 \\
\hline Race & 1 & 1 \\
African American & 1 & \\
Caucasian & 1 & 1 \\
Filipino & 2 & 3 \\
Hispanic & 1 & 1 \\
\hline Education & 2 & \\
High School Graduate & & \\
$1+$ yr. of college, No Degree & & \\
Associate Degree & & \\
Bachelor's Degree Master's Degree & & \\
Doctorate Degree & & \\
\hline
\end{tabular}

\section{Mothers as lead decision makers}

\section{Breastfeeding Decision Process}

For most mothers and fathers, the decision to breastfeed or formula feed their child did not take place prepartum. Many mothers shared that they informed their partners of their decision to breastfeed and partners were overwhelmingly supportive. One participant noted, "I did tell my husband. I did plan to breastfeed. He supported it. He always wants me and us to do what's best for our children. So, he was definitely on board. Very supportive. He just said whatever I decide". One father recalled, "Honestly the decision was all on my wife...My job [was to] support whatever she was comfortable with doing."

When asked if their partners' opinions about breastfeeding influenced their decision to breastfeed, most mothers shared that it did not. Many mothers reported that if a woman wanted to breastfeed, she would do so regardless of how anyone felt, including her partner. A mother went on to say that she believed it would help if the partner was supportive of the decision to breastfeed.

You gone [are going to] breastfeed regardless cause that's something that as a mother, or as a woman, you want to do. I think it's beneficial if your partner is supportive. That support makes a difference, but I don't think that's a determining factor.

\section{Support Provided by Fathers}

Although mothers were the lead decision makers, the support provided by fathers was helpful to the mother before and during the breastfeeding process. One mother recalled,
He would remind me to pump. He would sit there and keep [me] company while I pump[ed], and he would help me position them. He would be my coach. I mean he was $100 \%$ supportive. When I would get up at three in the morning he was up with me. He would try to help me position them, he would shove my nips in their mouth... He's a super mom.

Another mom responded by saying, "He helped a lot. He helped me through how to use the pump, how to help the baby latch on, how to hold the baby, how to tell when [the] breast is not as full as the other one, and [when to] switch over [to the other breast]". Fathers also recollected how they were able to provide support to mothers during breastfeeding. One father remembered,

I prepared the pump for her. I was bagging it [the milk] and putting it away. I was always helping getting the babies together. She was never just getting up by herself. I was always getting up with her to get the babies and help them latch on.

Another father shared, "You have to be able to do everything the momma does. Feeding, getting up, changing, bathing... [you] just got to be a team player. Make sure it's enough bottles when it's my turn to feed the baby". 
Most mothers reported that in the beginning stages of breastfeeding they were uncomfortable with public breastfeeding. To address their discomfort, mothers would breastfeed in the car ensuring that her breast and the baby were completely covered. Mothers who would breastfeed in public spaces would ask individuals around them if they were comfortable with her breastfeeding and adjusted her breastfeeding behaviors accordingly. Fathers also shared discomfort with public breastfeeding. In order to address this discomfort, fathers would use their bodies to shield mother and baby from the public view as this mother describes, "I think he was a little uncomfortable...on the train he was trying to butt up against [me] so that people couldn't see."

Across the sample, fathers were initially uncomfortable with public breastfeeding. This discomfort was not due to witnessing other mothers breastfeed, but was a protectiveness when strangers were present when their own partner breastfed. Fathers expressed that over time their discomfort decreased. One father said, "To me it was just a little different. It didn't bother me when it was someone else [breastfeeding]. But it was like it was my wife and that's the little protectiveness of me coming out."

\section{Prepartum Support of Breastfeeding}

Prepartum perspectives on breastfeeding were defined as the perspective fathers had about breastfeeding prior to the delivery of their own child. When asked about their partners' perspective of breastfeeding prepartum, many mothers could not answer because they had not discussed it with their partners before the baby was born. One participant noted, "So I don't know if we had an actual discussion. I think it was just moreso understood that, you know, we were going to breastfeed." Many of the fathers had no prior experience with breastfeeding so prior to the delivery of their child they had developed no opinion of it and they remained neutral on the topic of breastfeeding and were not for nor against it. Fathers who were born outside of the US were raised with the understanding that the purpose of breast was to breastfeed so their assumption was that their wives would breastfeed. One father born outside of the US recalled,

Well, when I was young living in [Name of Country], in [Name of City], it was common to see a mother on the bus or what have you, breastfeeding their child. It wasn't frowned upon, um you know that's the type of thing that few people have gotten a little over, over sensitive about it.

Other fathers reported that they believed breast milk would be healthier than formula for their babies.
Many parents discussed the benefits they observed in their child or among their family as a result of breastfeeding. Fathers reported the benefits they observed as a result of their child(ren) being breastfeed. These benefits included, but were not limited to breastmilk being healthy for the child, breastfeeding creating a stronger bond between the mother and child, saving the family money, and the child enjoying the time spent with the mother receiving comfort from being at the breast. One father stated,

He never once got sick. Never broke a fever, nothing; no colds, nothing... We think it's because she's giving him all his, her immune system to him, uh, through the breastfeeding. So that's why we're a little bit stronger on it now because we can see that it actually does help him.

\section{DISCUSSION}

The purpose of this study was to investigate the perspective fathers residing in Georgia had on breastfeeding and to explore if fathers influenced mothers' decision to initiate and continue exclusive breastfeeding for at least six months. Overall the results of this study showed that breastfeeding was common and that the reasons for initiating, continuing, supplementing or ceasing breastfeeding were not influenced by the father's perspective.

Data from this study showed that fathers of this educated demographic generally had no prepartum opinion of breastfeeding and deferred to their partner for decision making. The few fathers who had an opinion of breastfeeding prepartum reported a positive opinion informed by their cultural upbringing. Because most fathers had no prepartum opinion of breastfeeding, fathers did not strongly influence a mother's decision to initiate and continue exclusive breastfeeding for six months. However, having the father's support encouraged mothers to continue breastfeeding. Over time, many of the fathers developed a favorable opinion towards breastfeeding as a direct result of the perceived benefits it provided to their child(ren) and family.

Although fathers' views on breastfeeding did not influence mothers' decisions to breastfeed, mothers noted the importance of the fathers' postpartum support. This support ranged from providing the child with expressed breast milk, awakening in the middle of the night to support the mother, assisting with proper breastfeeding positions, coaching and encouraging mom when breastfeeding challenges arose, and assisting with preparation and cleanup of bottles and pump. 


\section{CONCLUSIONS}

The current study provides us with insight into the role that fathers have in the decision to breastfeed in Georgia. Despite the contributions of the findings, the study was not without limitations. Participant recruitment was slow and yielded a more highly educated demographic than intended. The researchers hypothesize that partner support for breastfeeding may vary by level of education or by cultural group. This is an area of future study.

This study found that those mothers of a higher education level were inclined to breastfeed their infant due to the many health benefits. Fathers either had no prepartum opinion about breastfeeding or they were explicitly supportive of breastfeeding due to their family traditions and/or cultures. Fathers developed an appreciation for breastfeeding after their babies were born. Mothers reported that though fathers' support of breastfeeding aided them during the breastfeeding process, it was not the determining factor.

\section{Acknowledgements}

The author would like to express her sincerest gratitude to Dr. Graybill and Dr. White for their continuous support during her thesis project. She would like to thank all study participants for their transparency. Lastly, she would like to thank her family: her significant other, daughter, and parents for their unfailing encouragement.

\section{References}

Ahluwalia, I., Morrow, B., and Hsia, J. Why do women stop breastfeeding? Findings from the Pregnancy Risk Assessment and Monitoring System. Pediatrics, 2005, 116(6): 14081412.doi: $10.1542 /$ peds.2005-0013

American Family Physicians. Breastfeeding (Policy Statement). AAFP. October 30, 2017.

https://www.aafp.org/about/policies/all/breastfeeding.html

American Academy of Pediatrics (AAP). Breastfeeding and the Use of Human Milk. Pediatrics, 2015, 115(2): 496-506. doi: 10.1542/peds.2004-2491

Arora S., M.C., Wehrer J., and Kuhn, P. Major Factors Influencing Breastfeeding Rates: Mother's Perception of Father's Attitude and Milk Supply. Pediatrics, 2000, 106(5).
Cai, X., Wardlaw, T., and Brown, D. Global Trends in Exclusive Breastfeeding. Int Breastfeed J, 2012, 7(1): 12 doi: 100.1186/1746-4358-7-12.

Centers for Disease Control and Prevention. Centers for Disease Control (CDC). Breastfeeding Report Card-United States 2016. Department of Health and Human Services. 2016. https://www.cdc.gov/breastfeeding/pdf/2016breastfeedingreport card.pdf

Cernadas, J., Graciela, N., Barrera, L., Martinez, A., and Garsd, A.Maternal and perinatal factors influencing the duration of exclusive breastfeeding during the first 6 months of life. J Hum Lact, 2003, 19(2): 136-144

Chang, J., and Chan, W. Analysis of factors associated with initiation and duration of breast-feeding: A study in Taitung Taiwan. Acta Paediatrica Taiwanica, 2003, 44(1): 29-34.

Freed, Gary, and J. Fraley. Effect of Expectant Mothers' Feeding Plan on Prediction of Fathers' Attitudes Regarding Breast- Feeding. Amer J Perinatol, 1993, 10.04:300-03.

Kessler, L. A., A. C. Gielen, M. Diener-West, and D. M. Paige. The Effect of a Woman's Significant Other on Her Breastfeeding Decision. J Hum Lact, 1995, 11.2:103-09.

Li, R., Fein, S.B., Chen, J., and Grummer-Strawn, L. M. Why mothers stop breastfeeding: mothers' self-reported reasons for stopping during the first year. Pediatrics, 2008, 122(S2):S69S76. doi: 10.1542/peds.2008-1315 Office of the Surgeon General (US). Barriers to Breastfeeding in the United States. 2016. https://www.ncbi.nlm.nih.gov/books/NBK52688/

Mannion, C.Hobbs, A., McDonald, S., and Tough, S. "Maternal Perceptions of Partner Support during Breastfeeding." Int Breastfeed J, 2013, internationalbreastfeedingjournal.biomedcentral.com/articles/1 0. 1186/1746-4358-8-4.

Meedya, S., Fathy, and K., Kable, A. Factors That Positively Influence Breastfeeding Duration to 6 Months: A Literature Review. Women Birth, 2010, 23(4): 135-145., doi:10.1016/j.wombi.2010.02.002.

Mueffelmann, Racine, Warren-Findlow, and Coffman, Perceived Infant Feeding Preferences of Significant Family Members and Mother's Intention to Exclusively Breastfeed. J Hum Lact, 2014, 31. Doi: $10.1177 / 0890334414553941$.

Sharma, M., and Petosa, R. Impact of expectant fathers in breastfeeding decisions. J AM Diet Assoc, 1997, 97(11): 1311-1313.

Unicef. Breastfeeding. Nutrition. UNICEF, July 29, 2015. http://www.unicef.org/nutrition/index_24824.html.

Unicef. Breastfeeding. Nutrition. UNICĒF, May 1, 2018. https://www.unicef.org/nutrition/index_breastfeeding.html

World Health Organization, Breastfeeding. WHO, December 14, 2011.

http://www.who.int/maternal_child_adolescent/topics/newborn/ nutrition/breastfeeding/en/

Q Les'Shon Irby, Emily Graybill, PhD, NCSP, and Cassandra White, PhD. Originally published in jGPHA (http://www,gapha,org/jgpha/) October 25, 2019. This is an open-access article distributed under the terms of the Creative Commons Attribution Non-Commercial No-Derivatives License (http://creativecommons,org/licenses/by/4.0/), which permits unrestricted use, distribution, and reproduction in any medium, provided the original work ("first published in the Journal of the Georgia Public Health Association...") is properly cited with original URL and bibliographic citation information. The complete bibliographic information, a link to the original publication on 